\title{
MODIS Brightness Temperature Change-based Forest Fire Monitoring
}

\author{
Fatemeh Parto $^{1,2^{*}}$, Mohammadreza Saradjian ${ }^{1}$, Saeid Homayouni $^{3}$ \\ 1 Remote Sensing Division, School of Surveying and Geospatial Engineering, College of Engineering, \\ University of Tehran, Tehran, Iran. \\ 2 Geodesy and Geomatics Faculty, K.N.Toosi University of Technology, Tehran, Iran. \\ 3 Centre Eau Terre Environnment, Insitut National de la Recherche Scientifique, Québec, Canada \\ * Corresponding authors: fparto.id@gmail.com
}

\begin{abstract}
Forests, as one of the most important natural resources in the world, are facing several challenges due to human activities and climate changes. The timely detection of forest fires plays an essential role in managing this wealth. Despite several well-developed fire detection methods, the detection of fires in early hours is still challenging. In this paper, we developed a new near-real-time hybrid method for fire detection that has high sensitivity to small and cold forest fires as well as less rate of false alarms. This method is based on detecting of both spatial and temporal changes. The change detection technique was used, and the identification of fire pixels was performed in the area with a significant change compared to the previous time. Since most of the false fire pixels had almost the same temperature in the images, they were masked. This mask allowed us to reduce the fire thresholds that lead to detect small fires. Furthermore, the omission and commission errors were minimized. This algorithm was applied to forty case studies in the north of Iran. The identified fire pixels were validated with ground observations collected by The Forests, Range and Watershed Management Organization (FRWMO) of Iran. Results show that the proposed algorithm was able to detect small and cool-case fires efficiently.
\end{abstract}

Keywords: Change Detection, Environment Management, Fire Detection, MODIS, Remote Sensing. 


\section{Introduction}

The Moderate Resolution Imaging Spectroradiometer (MODIS) is an instrument on the Terra and Aqua satellites which observes the Earth's surface every 1-2 days in 36 spectral bands. Instrument proficiency has been emphasized since the launch, and several fire detection methods based on MODIS imagery were proposed. These algorithms are mostly dependent on spatial hot spot detection using thermal infrared (TIR) bands.

Despite several algorithms for detecting fire, much remains to be considered to monitor the fire precisely. Kaufman et al. developed a MODIS algorithm for fire detection that set different thresholds for fires (Kaufman et al. 1998). Giglio et al. improved several aspects of this algorithm and proposed a contextual fire detection algorithm that has high sensitivity in detecting fire pixels (Giglio et al. 2003). MODIS Collection 4 Active Fire Products are based on this algorithm. Subsequently, this algorithm has been used in many studies (e.g., Chuvieco et al. 2008; Li et al. 2000; Cheng et al. 2013). For instance, Cheng et al. evaluated the MODIS product in the Yucatán Peninsula. They realized that omission errors were mainly caused by the potential fire threshold and reduced the daytime threshold to $305 \mathrm{~K}$ (Cheng et al. 2013). Moreover, examining the firing thresholds in the southeastern United States indicated that some fire pixels had brightness temperatures below the daytime potential fire threshold (Wang et al. 2006).

Consequently, Giglio et al. developed an enhanced algorithm for fire detection that overcomes some limitations in the previous algorithm (Giglio et al. 2016). In this algorithm, they identified areas that are susceptible to fires and reduced thresholds in these areas. The results indicated that this new fire detection algorithm had been improved compared to the previous one, with lower omission errors and false alarms. MODIS Collection 6 Active Fire Products are based on this developed algorithm. This algorithm has shown an efficient performance in several cases e.g. Furnacca et al. (2017); Abdollahi et al. (2018).

Wang used smoke bands and performed fire detection in the smoke area based on the smoke transmission radius. This algorithm can detect small and cold fires, especially at large scan angles, but it has a limitation in the non-smoke fire areas (Wang et al. 2009).

Although the efficient performance of the Giglio algorithm, still there are some limitations in detecting small fires. In the current study, we proposed a new forest fire detection algorithm based on Giglio et al., algorithm and change detection technique that could detect small fires with more accuracy. 


\section{Study area and data set}

\subsection{Study area}

About $8 \%$, or nearly 12.4 million hectares, of the total area of Iran, is covered by forests. Every year, there are devastating fires in Iran that damage the forest resources. The losses caused by forest fires are irrecoverable. The season of fire varies depending on humidity, wind, location and climatic conditions. In the northern part of Iran, we have most fires from August to the end of December. While most fires in the eastern part of the country happen in summer and winter. Golestan forest is located next to the Caspian Sea area. It has the most critical forest fields. Fifty-two fires were reported there in 1998. Based on (Ardakani et al. 2011.), 86.21\% of the fires detected by MODIS images occurred in cropland, grassland, and plain regions. Most of these fires have occurred in the eastern regions of the Caspian Sea. So we concentrated on this area.

\subsection{Satellite data}

The remote sensing data that used in this study consisted of MODIS Level 1B Radiance product (MOD021/MYD021) and the geolocation dataset (MOD03/MYD03). These data were obtained from the Earth Observing System Data Gateway, Land Processes Distributed Active Archive Centre (DAAC). The fires information was collected by The Forests, Range and Watershed Management Organization (FRWMO) of Iran and used to evaluate the results. Each forest fire case contains information on the initial and extinguishing time, location, and damaged area. The MOD03 was used to georeference the MOD021 Images. The atmospheric correction was performed using Flash software. Then the "bowtie" effect or overlap between MODIS scans corrected by the Wen algorithm (Wen et al. 2008). Later the radiances were converted to brightness temperatures by Planck's Radiation Law. Then, in order to reduce the stripping error, the de-striping tools in ENVI were used. Furthermore, the steps of the algorithm were done and then results validated with ground data.

\section{Proposed Algorithm}

In the proposed algorithm, based on the maximum sensitivity, MODIS 3.9 and $11 \mu \mathrm{m}$ channels were used to detect fire pixels in images. These channels are denoted by T3.9 and T11. The 250-1000 m resolution red and near-infrared channels were used to mask clouds, water and calculation of the Normalized Difference Vegetation Index (NDVI) index.

Our algorithm reached to high accuracy in four steps: first, masked out the water pixels based on the NDVI index. Second, removed the pixels which cause false alarms in boundaries by 
using dilation and closing morphological operation. Third, applied the change mask, which was performed, based on the pixel temperature difference of two consecutive images. This phase had a considerable enhancement in detecting small fires because it allowed reducing the fire thresholds. Finally, excluded the phase of false alarm rejection that removed some of the small and cold fires. The change mask phase removed most of the false alarm pixels due to the same situation of them in both images without omitting small fire pixels. The procedure of the proposed fire detection algorithm is described below.

\subsection{Cloud and Water Masking}

In this phase, channels 1, 2, 31 and 32 were used for the identification of cloud pixels. The pixel that satisfies the following tests is considered as cloudy pixels (Giglio et al. 2003.):

$$
\left(\rho_{0.65}+\rho_{0.85}>0.9\right) \text { or }\left(\mathrm{T}_{12}<265 \mathrm{~K}\right) \text { or }\left(\rho_{0.65}+\rho_{0.85}>0.7\right) \text { and }\left(\mathrm{T}_{12}<285 \mathrm{~K}\right)
$$

For the night image:

$$
T_{12}<265 K
$$

False alarms appeared in the cloud boundaries due to the low potential fire threshold. Accordingly, the dilation and closing of morphological operations were applied to the cloud mask to remove the cloud edge and gaps. Then, a water mask based on the NDVI index was performed on the images. Pixels with NDVI $<0.05$ were considered as water pixels.

$$
\mathrm{NDVI}=\frac{N I R-R}{N I R+R}
$$

\subsection{Change Detection}

Change detection is a technique to determine those pixels on images that have changed compared to the previous ones. Various environmental factors can change different aspects of pixels. This technique defines a threshold value to identify areas of "change" and "no change" pixels in the image.

In the proposed method, fire detection was performed on the pixels with noticeable brightness temperature changes over time that was recognized by a preliminary classification based on change detection. "No change" pixels are non-fire pixels, even if they have a high temperature, like hot desert pixels. In this phase, a change mask was applied to thermal bands between two images. Pixels with lower-threshold temperatures were classified as non-fire pixels. This threshold, which is denoted by $T d$ is set dynamically based on the average temperature difference of two images: 


$$
T_{d}=\frac{\bar{T}_{4}^{2}-\overline{T_{4}}}{3}
$$

This mask eliminated the pixels with small changes in brightness temperature. Many of pixels that constructed commission errors in previous works were removed at this stage because they almost had the same situation in both images. As a result, fire detection was applied to the pixels that have changed in thermal bands over time.

\subsection{Detection of Fire Pixels}

There are three logical stages through which fire pixels can be identified; i) detection of potential fire pixels, ii) absolute test, and iii) relative test. In the first step, a daytime pixel was identified as a potential fire pixel if T3.9> Td1, T3.9-T11> Td2 and $p_{.86}<0.3$.

Thresholds are identified based on $\operatorname{Td} 1$ and $\operatorname{Td} 2$. They set dynamically according to the brightness temperature of the pixels in the image. This way was suggested by (Zhukov et al. 2006). They calculated the thresholds based on the following equations:

$$
\begin{aligned}
& T d 1=\text { average }\left(T_{3.9}\right)_{j}+5 K \\
& T d 2=\text { average }(T 3.9-T 11)_{j}+5 k
\end{aligned}
$$

Where $j$ is the sample position in the range of $(0 \leq j \leq 1353)$. The pixels that passed these tests were classified as potential-fire pixels and considered in some tests to detect active fire pixels and those remained pixels were considered as non-fire pixels.

In the second step, the pixels containing active fires were identified. This threshold was set to the value 360/320 for day-/night-time, respectively (Kaufman et al. 1998).

$$
\begin{aligned}
& T_{4}>360 K \\
& T_{4}>320 K
\end{aligned}
$$

In the third phase, after detecting the spatial thermal variations, the relative tests were applied. The pixels around the potential fire pixel were used to estimate the background value. Within a window, the valid pixels are defined as those pixels which are located on the ground, do not contain cloud and subject to the following conditions:

$\mathrm{T} 3.9<315 \mathrm{~K}$ or $\mathrm{T} 3.9-\mathrm{T} 11<9.5 \mathrm{~K}$ for daytime, and $\mathrm{T} 3.9<305 \mathrm{~K}$ or $\mathrm{T} 3.9-\mathrm{T} 11>9.5 \mathrm{~K}$ for nighttime. Notably, we reduced the thresholds of this stage compared to (Giglio et al. 2003). The window starts as a $3 \times 3$ pixel and is maximized to $10 \times 10$ pixels as needed. In the conditions that at least four of the valid neighboring pixels could be identified, some measures were calculated. A summary of this process is presented in Table 1. 
Table1. Summary of the background pixels parameters (Giglio et al. 2003)

\begin{tabular}{cl}
\hline Parameter & Description \\
\hline$\overline{T_{4}}$ & mean of T3.9 for the valid neighboring pixels \\
\hline$\delta_{4}$ & mean absolute deviation of T3.9 for the valid neighboring pixels \\
\hline$\overline{T_{11}}$ & mean of T11 for the valid neighboring pixels \\
\hline$\delta_{11}$ & mean absolute deviation of T11 for the valid neighboring pixels \\
\hline$\overline{T_{\Delta T}}$ & mean of T3.9-T11 for the valid neighboring pixels \\
\hline$\delta_{\Delta T}$ & mean absolute deviation of T3.9-T11 for the valid neighboring pixels \\
\hline
\end{tabular}

167 In the relative tests, both the $3.9 \mu \mathrm{m}$ brightness temperature (T3.9) and the 3.9 and $11 \mu \mathrm{m}$ brightness temperature difference $(\Delta \mathrm{T})$ was used. Relative thresholds were determined based on the natural changes in the background. These tests are defined as follows:

$\Delta T>\Delta T+3.5 \sigma_{\Delta T}(2)$

$172 \Delta T>\Delta T+6 K$

$173 T_{4}>\overline{T_{4}}+3 \sigma_{4}$

$174 \quad T_{11}>\overline{T_{11}}+\sigma_{11}$ $\sigma_{11}>5 k$

A daytime pixel is classified as a fire pixel if (test (1) is true) or (tests (2)-(4) are true and one of the tests of (5) or (6)). Otherwise, it is classified as non-fire.

178 A night-time fire pixel is classified as fire if test (1) is true or tests (2)-(4) are true; otherwise, 179 it is classified as non-fire (Giglio et al. 2003).

180 The schematic illustration of the proposed algorithm is shown in Fig. 1. 


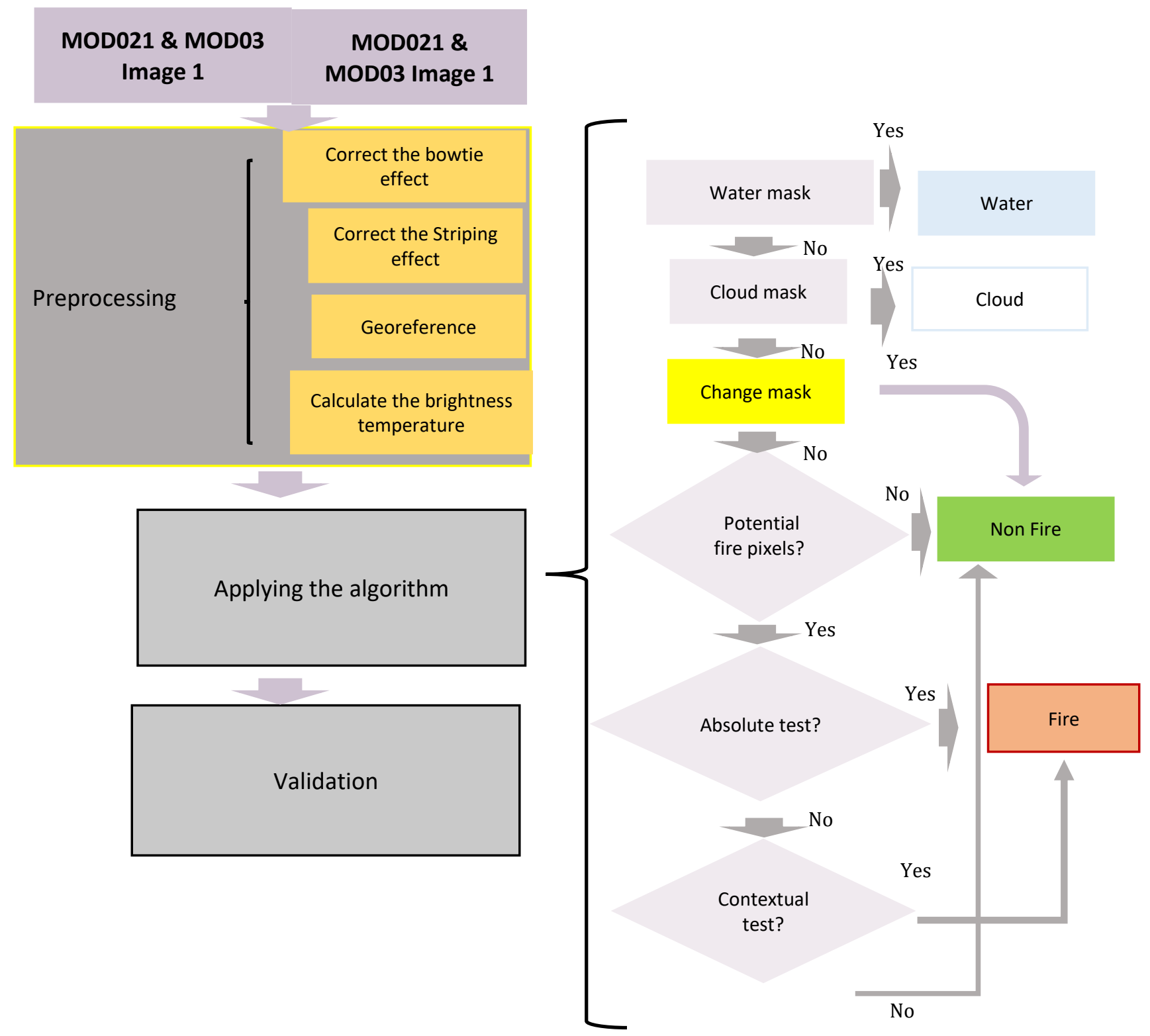

Fig. 1. Flowchart of the proposed fire detection algorithm

\section{Result and Discussion}

In this paper, we proposed a new hybrid fire detection method based on both spatial and temporal changes. Fuel type and weather conditions have a significant influence on predisposing an area. Consequently, some improvements must be made for small fire detection on a regional scale and the potential fire threshold should be defined low enough. However, a lower threshold may increase the number of false alarms. Moreover, the false alarm rejection step in Giglio et al. algorithms eliminates some small fires as well.

To date, most of the exiting omission error reduction techniques increase commission errors and conversely, furthermore the fire detection algorithms are mostly based on analyzing the 
spatial variation. While investigating temporal variation can significantly enhance the accuracy of detection of fire pixels. In our work, this point was considered. It can be seen clearly that when a fire occurs in some pixels, their temperature has been changed significantly compared to the previous times. So a change mask was exploited in the proposed algorithm, and fire pixels were detected among the pixels that had a temperature variation. In particular, the proposed algorithm looks for a pixel that is hotter than both its neighborhoods and itself over time. This technique removed most of the false alarms without deleting fire pixels. Furthermore, this step had a significant enhancement for the small fire detection because the fire thresholds could be reduced without increasing the number of false alarms, and both commission and omission errors were minimized.

This algorithm was applied to forty case studies in the eastern regions of the Caspian Sea and validated by data collected by the Forests, Range and Watershed Management Organization (FRWMO) of Iran. The number of false alarms before and after applying the change mask for each case study is shown in Fig. 2.

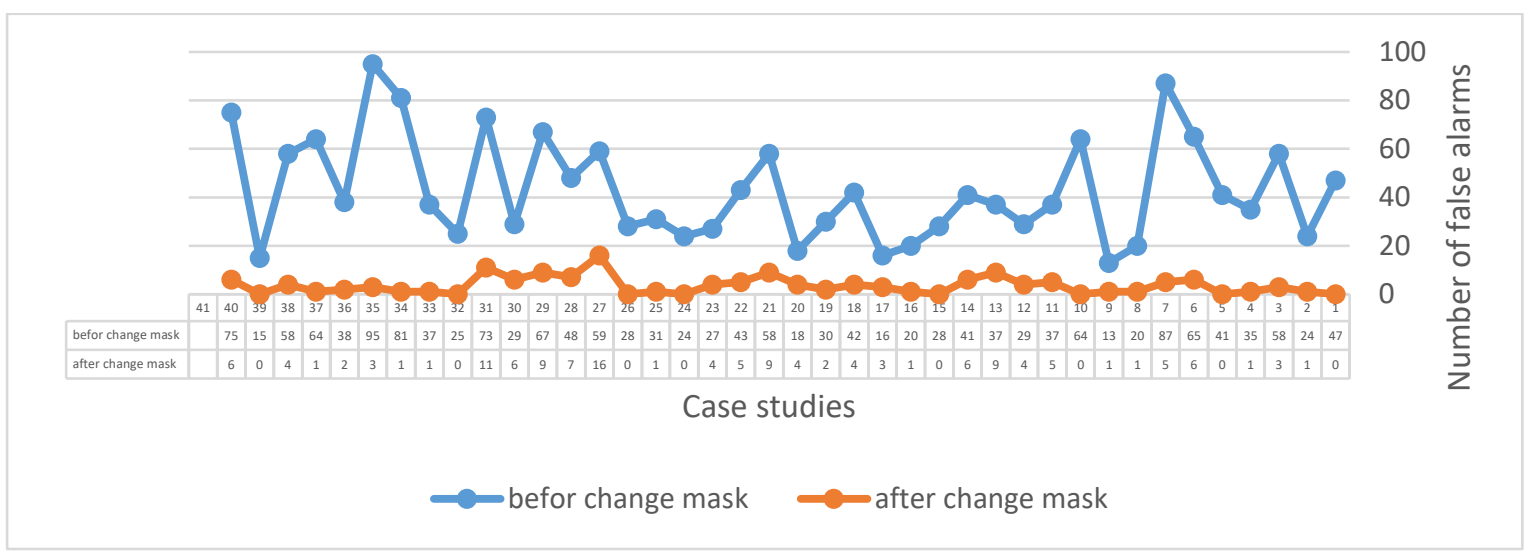

Fig. 2. Number of false alarms before and after change mask for each case study

As an example, the number of false alarms for the first and second images is presented in table 2 .

Table. 2. Effect of each mask on the number of false alarms in the first and second case study

\begin{tabular}{llcc}
\hline & & Before applying the mask & After applying the mask \\
\hline \multirow{2}{*}{ Water Mask } & Image1 & 97 & 47 \\
\cline { 2 - 4 } Change Mask & Image2 & 51 & 24 \\
\cline { 2 - 4 } & Image1 & 47 & 0 \\
\hline
\end{tabular}


217 The proposed algorithm detected 39 fires of case studies and some false alarms. Next, the 218 Giglio algorithm was applied to these fire images. This algorithm only traversed 32 cases; furthermore, the number of false alarms was less than the proposed algorithm in 15 cases. In these cases, the second image was checked out, and by replacing the more appropriate one, the number of false alarms was decreased. The probability of fire detection in the proposed algorithm is strongly dependent on the condition of the second image. Different images as the second image with different time intervals were used. Results demonstrate that when the time interval between the two images in the change mask was increased, the number of false alarms was grown up. Consequently, to achieve high accuracy in this algorithm, the weather condition in the second image must be similar to the main image and missing data and cloud pixels should be minimal.

Examples of detected fires in the first and second case studies are shown in Fig.3.
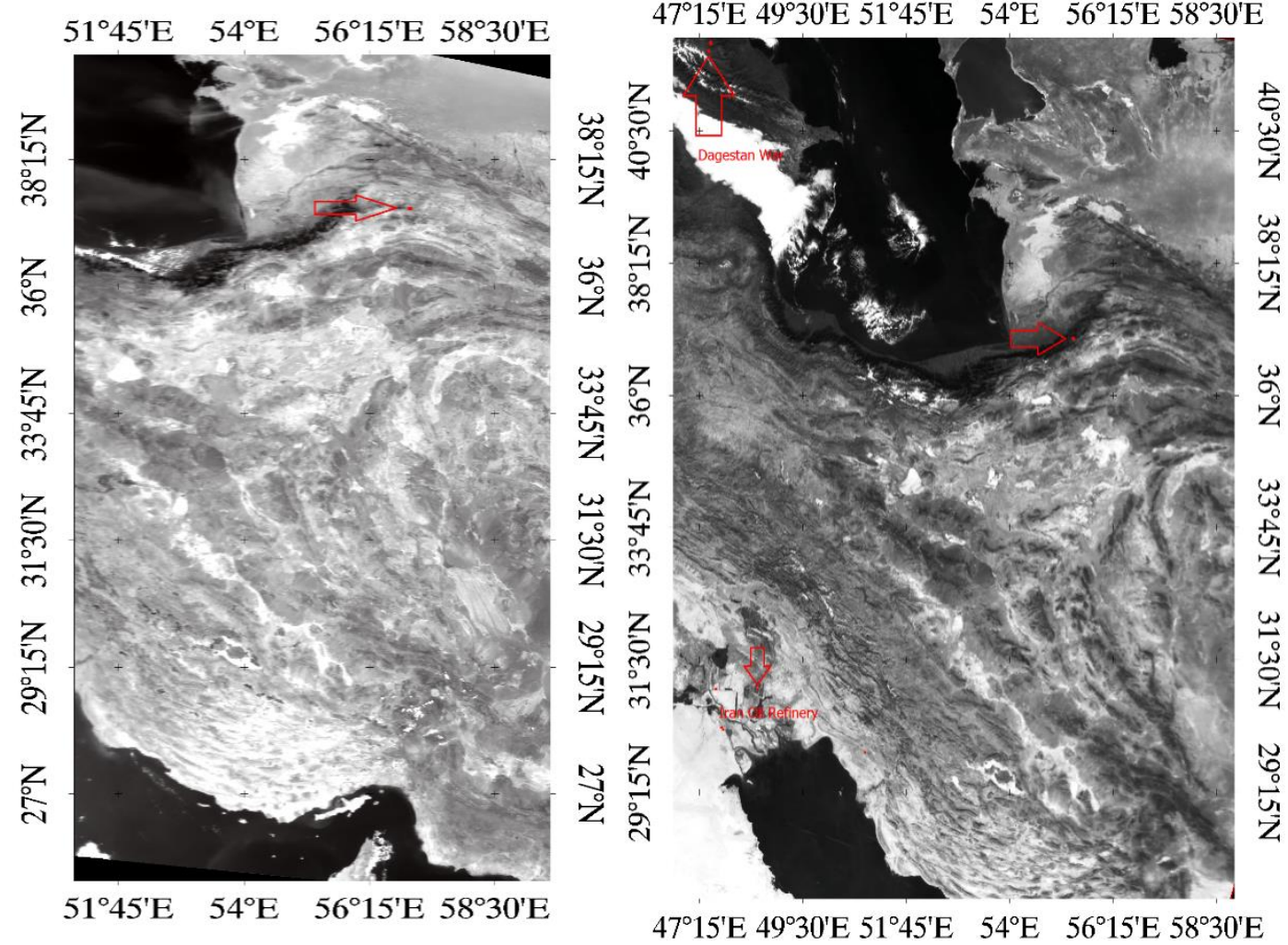

Fig. 3. An example of detected fires in the first and second case study

\subsection{Accuracy Assessment}

In order to evaluate this algorithm, Reference-based accuracy, Omission Error, Map-based accuracy and Commission Error were calculated:

- Reference-based accuracy or Producer's accuracy is obtained by the ratio of the number of correct pixels into the total number of pixels in a class. 
Producer's accuracy = classified pixels in the specified class/ground reference pixels in this class

- Omission Error means c pixel that should have been included in the class:

$$
\text { Omission Error }=1 \text { - Producer's accuracy }
$$

- Map-based accuracy or User's accuracy that determining the percentage of correct predictions:

User's accuracy = correctly classified pixels as a specified class /total of classified pixels as the this specified class

- Commission Error means including a pixel in a class when it had to be removed:

Commission Error = 1 - User's accuracy

These parameters and values obtained from the proposed algorithm and the Giglio algorithm are presented in table 3 .

Table. 3. Effect of each mask on the number of false alarms in the first and second case study

\begin{tabular}{ccc}
\hline Parameter & First Case Study & Second Case Study \\
\hline Producer's accuracy & $93.5 \%$ & $80 \%$ \\
\hline Omission Error & $2.5 \%$ & $20 \%$ \\
\hline User's accuracy & $89 \%$ & $95.5 \%$ \\
\hline Commission Error & $11 \%$ & $4.5 \%$ \\
\hline
\end{tabular}

\section{Conclusion}

We have developed an appropriate platform for small and cold fire detection. One of the crucial reasons for false alarms in previous works was water pixels. Consequently, in the currently proposed algorithm, we masked out the water pixels using the NDVI index. After that, a dilation and closing morphological operation were used to remove the pixels, which cause false alarms in boundaries. The result showed that the proposed algorithm had no false alarm from water pixels in the case studies. The milestone of this study was to identify the hot spots based on both temporal and spatial changes. A change mask was applied to the fire image, which was performed based on the difference between thermal bands of two images. This mask allowed us to reduce the fire thresholds to a regional low and cold fire temperature and had a considerable enhancement in detecting fires in early hours, so the omission errors were minimized.

Moreover, our algorithm does not need to false alarm rejection step which is considered as one of the main phases in MODIS Active Fire algorithms and omitted some of the small and cold fires, because the change mask removed most of these false alarm pixels without deleting fire pixels since they had the same situation in both images without omitting small fire pixels. Therefore, It leads to a decreasing in commission errors. Together, our results indicated that the proposed method had a good improvement for identifying fires in the first hours to prevent it from spreading and had a notable improvement to minimize commission and omission errors.

\section{Acknowledgment}


We thank Forests, Range and Watershed Management Organization (FRWMO) of Iran for trooping ground observations and Earth Observing System Data Gateway, Land Processes Distributed Active Archive Centre (DAAC) for providing MODIS data.

\section{References}

Abdollahi, M., Islam, T., Gupta, A., \& Hassan, Q. (2018). An Advanced Forest Fire Danger Forecasting System: Integration of Remote Sensing and Historical Sources of Ignition Data. Remote Sensing, 10(6), 923. https://doi.org/10.3390/rs10060923

Ardakani, A. S., Zoej, M. J. V., Mohammadzadeh, A., \& Mansourian, A. (2011). Spatial and Temporal Analysis of Fires Detected by MODIS Data in Northern Iran From 2001 to 2008. IEEE Journal of Selected Topics in Applied Earth Observations and Remote Sensing, 4(1), 216-225. https://doi.org/10.1109/JSTARS.2010.2088111

Cheng, D., Rogan, J., Schneider, L., \& Cochrane, M. (2013). Evaluating MODIS active fire products in subtropical Yucatán forest. Remote Sensing Letters, 4(5), 455-464. https://doi.org/10.1080/2150704X.2012.749360

Chuvieco, E., Giglio, L., \& Justice, C. (2008). Global characterization of fire activity: toward defining fire regimes from Earth observation data. Global Change Biology, 14(7), 1488-1502. https://doi.org/10.1111/j.1365-2486.2008.01585.x

Fornacca, D., Ren, G., \& Xiao, W. (2017). Performance of Three MODIS Fire Products (MCD45A1, MCD64A1, MCD14ML), and ESA Fire_CCI in a Mountainous Area of Northwest Yunnan, China, Characterized by Frequent Small Fires. Remote Sensing, 9(11), 1131. https://doi.org/10.3390/rs9111131

Giglio, L., Descloitres, J., Justice, C. O., \& Kaufman, Y. J. (2003). An Enhanced Contextual Fire Detection Algorithm for MODIS. Remote Sensing of Environment, 87(2-3), 273-282. https://doi.org/10.1016/S0034-4257(03)00184-6

Giglio, L., Schroeder, W., \& Justice, C. O. (2016). The collection 6 MODIS active fire detection algorithm and fire products. Remote Sensing of Environment, 178, 31-41. https://doi.org/10.1016/J.RSE.2016.02.054

Kaufman, Y. J., Justice, C. O., Flynn, L. P., Kendall, J. D., Prins, E. M., Giglio, L., et al. (1998). Potential global fire monitoring from EOS-MODIS. Journal of Geophysical Research: Atmospheres, $103(\mathrm{D} 24)$, 32215-32238. https://doi.org/10.1029/98JD01644@10.1002/(ISSN)2169-8996.EOSAM1

Li, Z., Kaufman, Y. J., Ichoku, C., Fraser, R. H., Trishchenko, A. P., Giglio, L., et al. (2000). A Review of AVHRR-based Active Fire Detection Algorithms: Principles, Limitations, and Recommendations. https://www.semanticscholar.org/paper/A-Review-of-AVHRR-basedActive-Fire-Detection-and-Li-Kaufman/dbed83d5a47f63f377c9c3dbf714b57225f654ca. Accessed 6 October 2019

Movaghati, S., Samadzadegan, F., \& Azizi, A. (2008). A COMPARATIVE STUDY OF THREE ALGORITHMS FOR FOREST FIRE DETECTION IN IRAN. https://www.semanticscholar.org/paper/A-COMPARATIVE-STUDY-OF-THREEALGORITHMS-FOR-FOREST-MovaghatiSamadzadegan/c904da8dc83e62a44bc3b494cfbc2fcfd35c4e0e. Accessed 6 October 2019

Wang, W., Hao, X., Liu, Y., \& Sommers, W. T. (2007). An improved algorithm for small and cool fire detection using MODIS data: A preliminary study in the southeastern United States. Remote Sensing of Environment, 108(2), 163-170. https://doi.org/10.1016/J.RSE.2006.11.009

Wang, W., Qu, J. J., Hao, X., \& Liu, Y. (2009). Analysis of the moderate resolution imaging spectroradiometer contextual algorithm for small fire detection. Journal of Applied Remote Sensing, 3(1), 031502. https://doi.org/10.1117/1.3078426

Wen, X. (2008) . A New Prompt Algorithm For Removing The Bowtie Effect Of Modis L1b Data. Commission III, WG III/1. 
Zhukov, B., Lorenz, E., Oertel, D., Wooster M., \& Roberts, G. (2006). Spaceborne Detection And Characterization Of Fires During The Bi-Spectral Infrared Detection (BIRD) Experimental Small Satellite Mission (2001-2004). Remote Sensing of Environment, 100, 29-51. 